\title{
Genetic Diversity Analysis of Ethiopian Yam (Dioscorea spp) from Different Geographic Origin Using Simple Sequence Repeat
}

\author{
Atnafua Bekele ${ }^{1}$, Endashaw Bekele ${ }^{2}$ \\ ${ }^{1}$ South Agricultural Research Institute, Hawassa Agricultural Research Centre, Hawassa, Ethiopia \\ ${ }^{2}$ Addis Ababa Universities, Microbial, Cellular and Molecular Biology Department, Addis Ababa, Ethiopia
}

Email address:

atnaf_ruth@yahoo.com (A. Bekele), endashawbw@gmail.com (E. Bekele)

To cite this article:

Atnafua Bekele, Endashaw Bekele. Genetic Diversity Analysis of Ethiopian Yam (Dioscorea spp) from Different Geographic Origin Using Simple Sequence Repeat. Journal of Plant Sciences. Vol. 9, No. 3, 2021, pp. 96-106. doi: 10.11648/j.jps.20210903.14

Received: September 16, 2020; Accepted: May 26, 2021; Published: June 21, 2021

\begin{abstract}
Yam (Dioscorea spp L.) is one of root and tuber crops grown in Ethiopia as source of food and income. It is cultivated mainly in south, southwest, and western part of the country. Two hundred ten yam accessions from ten different geographic origins of major growing areas of the country were used in this study. The sprouted tubers of some accessions were received from research centers where others were directly collected from farmers' fields during early March, 2010. The collected yam genotypes were planted in complete randomized block design at two research sites (namely at Hawasa and Wonago) which are found under South Agricultural Research Institute in the end of April 2010. All important cultural practices such as staking, weeding and irrigation were done starting from planting till harvesting. Yam leaf samples with of different origin were observed separately using twenty SSR markers. A similarity matrix derived UPGMA cluster analysis based on geographical sites showed nine distinct clustering groups which indicated presence of relationship between genetic distances to that of geographical distance in most of clustering groups. These cluster grouping is supported by Analysis of Molecular Variance (AMOVA) that indicated presence of significance genetic variation of 210 yam accessions within and among ten collection sites.
\end{abstract}

Keywords: Yam, Genetic Diversity, Geographic Distance, SSR

\section{Introduction}

Ethiopia has diverse biological wealth that includes plants, animals, and microbial species. The crop diversity of the country is very high due to the existence of diverse farming systems, socioeconomics, cultures and agro-ecologies. There are several indigenous cultivated or semi-cultivated root and tuber crops in Ethiopia. These crops have an important place in the diet of the population [1]. Yam is among the most important tuber crops in the country, especially in humid areas where there is heavy year round precipitation [2]. It is cultivated in South, South Eastern and South Western parts of the country across a range of agro-ecologies as the main co-stable food stuffs. It is known by different vernacular names in different locations such as Boye, by Dauro people; Kocho or Wocheno, Oromo people; Bohe, Wolayita people; Kuso, Yem and Sidama people: Boina, [3].
It is a preferred food and plays a major role in food security. It has high price at market compared to other root and tuber crops produced in the country. Hence, it is a good source of income for producing farmers.

Several species of Dioscorea grow in different parts of Ethiopia but not all are edible. Eleven described Dioscorea species, (both wild and cultivated), are found in the country indicating presence of wide diversity of the species. The species D. alata $\mathrm{L}$ is not indigenous to Ethiopia and its center of origin is Southeast Asia. In Ethiopia, D. alata it is sterile and grows only in cultivation. Predominantly six yam species are grown by farmers in the country. These are D. rotundata D. bulbifera, D. abyssinica, D. cayenensis, D. praehensilis, and D. alata [4].

The present study is conducted with more yam accessions from Gamogofa and Wolita regions based on earlier recommendations and tried to cover more geographic regions 
of major growing areas of the country (Table 3). It evaluates the genetic diversity and relationships of 210 yam accessions across in ten geographic area of Ethiopia using Simple Sequence Repeat Poly Acrylamaide Gel Electrophoresis based analysis.

\section{Objective of the Study}

The major objectives of this research is to determine the degree of genetic diversity and the relationship among and within Ethiopian yam germplasms collected from ten major growing areas of the country.

\section{Methodologies Used in the Study}

\subsection{DNA Extraction}

Young and clean leaves were collected and dried in silca-gel. These dried samples were grounded with sterile pestle and mortar with addition of liquid nitrogen. $0.2 \mathrm{~g}$ of pulverized leaf sample was used to isolate genomic DNA using modified triple Cetyl Trimethyl Ammonium Bromide (CTAB) extraction technique [5]. The quality and yield of DNA was carried out by running $3 \mu \mathrm{l}$ of freshly extracted genomic DNA samples on $1 \%$ agarose gel stained with $3 \mu$ of ethidium bromide, and visualized under an ultraviolet transiluminator in Genetic Research Laboratory of Addis Ababa University, Addis Ababa, Ethiopia (Figure 1). The quality and concentration of all DNA samples were determined using NanoDrop spectrophoto meter (NanoDrop Technologies, Wilming Ton, DE) at the International Institute of Tropical Agriculture (IITA), Ibadan. The NanoDrop uses $2 \mu \mathrm{l}$ of diluted DNA samples (100 $\mu \mathrm{l} \mathrm{TE})$ with a sensitivity range from 5ng to $3700 \mathrm{ng}$. Absorbance at $260 \mathrm{~nm}$ (A260) is measured for each DNA samples to determine the DNA quantity. Nucleic acids and proteins have absorabance maxima at 260 and $280 \mathrm{~nm}$ respectively. A260 measurments are quantitatitaive for relatively pure DNA preparations in microgram quanities. However the absorbance wave length ratio (260/280) was used to determine the quality of DNA. The ratio of 260 and $280 \mathrm{~nm}$ used as an indicator of DNA purity. The absorbance wave length ratio (260/280) of the genomic DNA was between 1.8 to 2.0 .

\subsection{PCR Amplification}

All the DNA samples were diluted to a standard concentration of $25 \mathrm{ng} / \mu \mathrm{l}$ with ultra pure water for PCR amplification. Polymerase chain reactions (PCRs) were carried out in a total volume of $10 \mu \mathrm{l}$, containing $2 \mu \mathrm{l}$ of $25 \mathrm{ng}$ of genomic DNA, $1 \mu \mathrm{l}$ of $5 \mathrm{mM}$ forward primer, $1 \mu \mathrm{l}$ of $5 \mathrm{mM}$ of reverse primer, $1 \mu 110 \mathrm{X} \mathrm{NH} 4$ reaction buffer, $0.4 \mu \mathrm{l}$ of 50 $\mathrm{mM} \mathrm{MgCl}_{2}, 0.4 \mu \mathrm{l}$ of $5 \mathrm{mM} \mathrm{dNTP}$, and $0.06 \mu \mathrm{l}(5 \mathrm{u} / \mu \mathrm{l}$, Bioline) Biotaq ${ }^{\mathrm{TM}}$ DNA polymerase. Touchdown PCR was performed using Applied Biosystems verti 96 well thermal cycler. In this program the anealing temprature was gradually decreased by $1^{0} \mathrm{C}$ during the cycling program in order to favour only specific product amplification. The PCR cycle consisted of initial denaturation at $95^{\circ} \mathrm{C}$ for 2 minute followed by 35 cycles for $65^{\circ} \mathrm{C}$ anealing for 20 seconds then with $1^{\circ} \mathrm{C}$ reduction in temprature per cycle for 10 cycles and the last extension step was $72^{\circ} \mathrm{C}$ for 5 minute. The PCR products were storred at $4{ }^{\circ} \mathrm{C}$ until used. Touchdown PCR is a modification of PCR in which the intial annealing temprature is higher than the optimal and is gradually reduced over subsequent cycles until the Tm temprature or "touchdown temprature" is reached. The annealing temprature is decreased $1^{0} \mathrm{C}$ every other cycle to approx $10^{\circ} \mathrm{C}$ below the calculated $\mathrm{Tm}$ to permit exponential amplification [6].

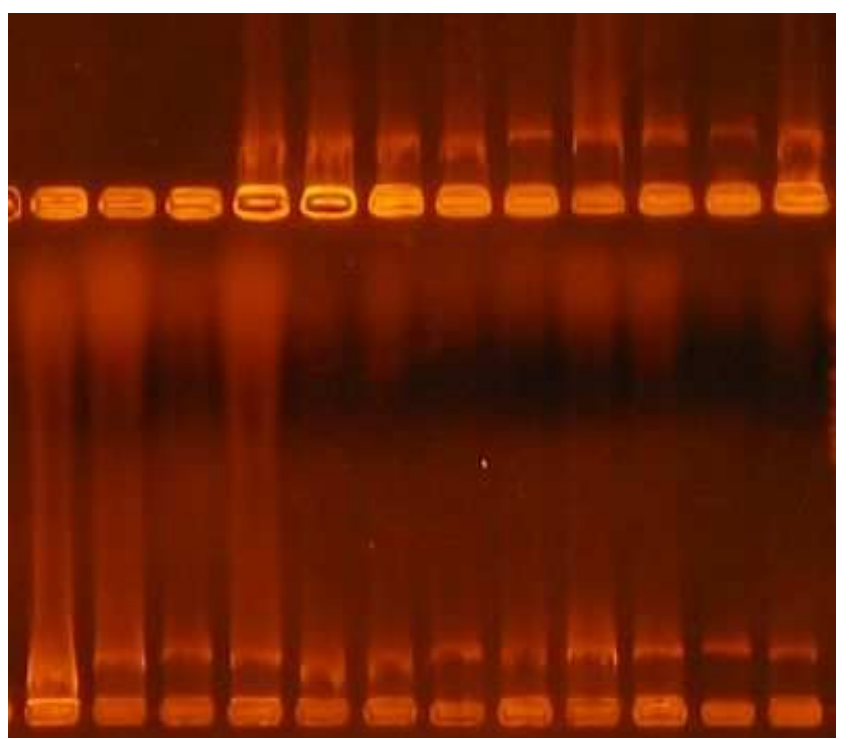

Figure 1. Test gel of some diluted DNA samples extracted from Ethiopian Yam (Dioscorea spp) collections.

\subsection{Microsatellite Markers and Detection of PCR Products}

In this procedure, two cleaned balanced plates were taken and at the edges of the plates, polyacrylamide gel was poured immediately. After the gel become polymerized, the plates were fixed to the tanker and 1x TBE was added at its upper side. The tanker was connected to a circute and left until it become warm. The denatured PCR products through formamide dye were loded in to gel approximetly with amount of $2.5 \mathrm{ul}$ inside each well. This was allowed to run at 80 Watt for one and half hours to two and half hours according to the molecular weight of the primers. Then after, the gel was subjected to fixing solution for 20-30 minutes, staining solution for 30 minutes, distilled water for seconds, developing solution and it was shaked by hand until the bands were visable. At end the bands were fixed using fixing solution.

\subsection{Details of Germplasm Studied and Micro Satellite Primers}

Two hundred fifty eight yam accessions which were collected from ten major growing areas of Ethiopia were considered for this study. A total of 20 (thirteen DNA microsatellite markers and seven EST derived) markers were used in this study, of which 14 (eleven DNA microsatellite 
markers and three EST derived) microsatellite markers resulted in allelic bands (Table 1). However, among these twelve (ten DNA microsatellite markers and two EST derived) microsatelite markers were gave clear bands for all studied germplasms and these SSR markers were taken for diversity analysis study (Table 2).
Loading of the DNA samples on Polyacrylamide Gel were carried in two different phases. First 96 denatured DNA samples were loaded on plate 1 and depending on the expected size of the PCR products with 20-30 minutes gap the second denatured DNA samples were loaded on plate 2 . The rest 66 samples were loaded on other third plate.

Table 1. The list of microsatellite primers observed in this study.

\begin{tabular}{llllllllll}
\hline No. & SSR Primers & Plate1 & Plate2 & Plate3 & No. & SSR Primers & Plate1 & Plate2 & Plate3 \\
\hline 1 & YM13 & $*$ & $*$ & $*$ & 11 & D9 & $*$ & $*$ & $*$ \\
2 & Da1C12 & $*$ & $*$ & $*$ & 12 & Dpr3F12 & $*$ & $*$ & X \\
3 & Da1A01 & $*$ & $*$ & $*$ & 13 & Dpr3B12 & $*$ & & $*$ \\
4 & Dpr3D06 & $*$ & $*$ & $*$ & 14 & D95 & X & X & $*$ \\
5 & Da1F08 & $*$ & $*$ & $*$ & 15 & D58 & X & X & $*$ \\
6 & DA1D08 & $*$ & $*$ & $*$ & 16 & D25 & X & X & $*$ \\
7 & Dab2C12 & $*$ & $*$ & $*$ & 17 & YM26 & X & X & X \\
8 & Ym30 & $*$ & $*$ & $*$ & 18 & DPR3F04 & $*$ & $*$ & $*$ \\
9 & D83 & $*$ & $*$ & $*$ & 19 & D91 & $*$ & $*$ & X \\
10 & D55 & $*$ & $*$ & $*$ & 20 & Dab2C05 & $*$ & & $*$ \\
\hline
\end{tabular}

Note:- $*=$ SSR primers showed amplified products, $\mathrm{x}=$ SSR primer did not work, Plate $1 \&$ Plate $2=$ Each 96 samples were loaded Plate $3=66$ DNA samples were loaded

Table 2. Primer sequences (forward and reserve) used in the SSR analyses and their respective repeat motif, annealing temperature (Ta), Size (bp) and number of alleles per locus $(A)$.

\begin{tabular}{|c|c|c|c|c|c|}
\hline Locus & Primer sequence (5'-3') & Repeat Motif & Size (bp) & Ta $\left({ }^{\circ} \mathrm{C}\right)$ & $\mathbf{A}$ \\
\hline DalC12 & $\begin{array}{l}\text { F:GCCTTTGTGCGTATCT } \\
\text { R:AATCGGCTACACTCATCT }\end{array}$ & $(\mathrm{GT}) 9$ & 163 & & 5 \\
\hline Ym30 & $\begin{array}{l}\text { F:GGTCCTCTTCTATCCCAACAA } \\
\text { R:CACGTATTAACTCCATCATCC }\end{array}$ & & & & 4 \\
\hline Da1Do8 & $\begin{array}{l}\text { F:GATGCTATGAACACAACTAA } \\
\text { R:TTTGACAGTGAGAATGGA }\end{array}$ & $(\mathrm{CA}) 8$ & 335 & 51 & 4 \\
\hline Dpr3Fo4 & $\begin{array}{l}\text { F:AGACTCTTGCTCATGT } \\
\text { R:GCCTTGTTACTTTATTC }\end{array}$ & $(\mathrm{AG}) 15$ & 128 & 51 & 10 \\
\hline D55 & $\begin{array}{l}\text { F:TGGACTAACGTGGTGTAGG } \\
\text { R:CTAACAACACACACACGGG }\end{array}$ & & & & 2 \\
\hline D83 & $\begin{array}{l}\text { F:AGCTGAGATGGGAGGATCAA } \\
\text { R:AGGAGGAGGTGGAGGACTTC }\end{array}$ & & & & 7 \\
\hline Da1Ao1 & $\begin{array}{l}\text { F:TATAATCGGCCAGAGG } \\
\text { R:TGTTGGAAGCATAGAGAA }\end{array}$ & GT) 8 & 204 & 51 & 6 \\
\hline dab2C12 & $\begin{array}{l}\text { F:GCCTTTGTGCGTATCT } \\
\text { R:AATCGGCTACACTCATCT }\end{array}$ & $(\mathrm{AC}) 8$ & 163 & & 4 \\
\hline Da1Fo8 & $\begin{array}{l}\text { F:AATGCTTCGTAATCCAAC } \\
\text { R:CTATAAGGAATTGGTGCC }\end{array}$ & (TG)13 & 177 & 51 & 6 \\
\hline YM13 & $\begin{array}{l}\text { F:TTCCCTAATTGTTCСТCTTGTT } \\
\text { R:GTCCTCGTTTTCCCTCTGTGT }\end{array}$ & & & 58 & 5 \\
\hline Dpr3D06 & $\begin{array}{l}\text { F:ATAGGAAGGCAATCAGG } \\
\text { R:ACCCATCGTCTTACCC }\end{array}$ & (GA)15 & 151 & 51 & 6 \\
\hline Dpr3B12 & $\begin{array}{l}\text { F:CATCAATCTTTCTCTGCTT } \\
\text { R:CCATCACACAATCCATC }\end{array}$ & (TG) 8 & 135 & 51 & 8 \\
\hline
\end{tabular}

Note:- $\mathrm{F}=$ forward primer sequence $\mathrm{R}=$ reverse primer sequence

\subsection{Data Scoring and Statistical Data Analysis}

Microsatelites (SSR) scores were done for all 258 DNA samples. The score is converted into binary data by presence (1) or absence (0) of the selected fragment (respective allele in the case of SSR loci).

Among 258 yam accession, 48 accessions were duplicates which were identical at all SSR loci. During the analysis one acession was selected from each duplicates to represent the genotype and hence 210 yam land races were considered for the statistical anlysis (Table 3)

Analysis of Molecular Variance was computed following detection of PCR products and data scoring using the computer program Gen Alex [7].

The POPGENE 1.32 program [8] was used to calculate the genetic diversity parameters: allele frequencies at each locus the number of observed alleles, the number of effective alleles, Shannon information index (I) and gene diversity $(\mathrm{H})$. The distance matrixes and the un-weighted Pair Group Method with arithmetic mean (UPGMA) [8] 
were generated using Free Tree 0.9.1.50 Software and the distance tree was then viewed using TREEVIEW 0.9.1.50 Software of Tree un-rooted and slanted methods for species based and site based populations respectively [9].
The patterns of variation among yam population on $2 \mathrm{D}$, a principal coordinated analysis (PCA) was examined using NTSYS-pc version 2.2 based on Nei's unbiased genetic distance [10 and 11].

Table 3. List of Ethiopian yam collection from 10 different major growing areas of the country.

\begin{tabular}{|c|c|c|c|c|}
\hline SN & Accession number & Zone & Location & Altitude(Local name) \\
\hline 1 & Sil & Gamogofa & Kucha & 1500 (Bunne3) \\
\hline 2 & $\mathrm{Si} 2$ & Sidama & Dalle & 1940 (Gellawcho-1) \\
\hline 3 & $\mathrm{Si} 3$ & Sidama & Dalle & 1940 (Gelawcho-2) \\
\hline 4 & $\mathrm{Si} 4$ & Sidama & Dalle & 1940 (Midasho-1) \\
\hline 5 & $\mathrm{Si} 5$ & Sidama & Dalle & 1940 (Midasho-2) \\
\hline 6 & $\mathrm{Si6}$ & Sidama & Dalle & 1940 (Ouwisho-1) \\
\hline 7 & $\mathrm{Si} 7$ & Sidama & Dalle & 1940 (Ouwisho-2) \\
\hline 8 & $\mathrm{Si} 8$ & Sidama & Dalle & 1940 (Ouwisho-3) \\
\hline 9 & $\mathrm{Si} 9$ & Sidama & Dalle & 1940 (Wendu-1) \\
\hline 10 & Si10 & Sidama & Dalle & 1940 (Adameado-1) \\
\hline 11 & Sil1 & Sidama & Dalle & 1940 (Adameado-2) \\
\hline 12 & Si12 & Sidama & Dalle & 1940 (Wendu-2) \\
\hline 13 & Si13 & Sidama & Dalle & 1940 (Wendu-3) \\
\hline 14 & Si14 & Sidama & Dalle & 1940 (Wendu-4) \\
\hline 15 & Si15 & Sidama & Dalle & 1940 (Genticha-1) \\
\hline 16 & Sil6 & Sidama & Dalle & 1940 \\
\hline 17 & Si17 & Sidama & Dalle & 1940 (Genticha-2) \\
\hline 18 & Sil8 & Sidama & Dalle & 1940 (Genticha-3) \\
\hline 19 & Si19 & Sidama & Dalle & 1940 (Genticha-4) \\
\hline 20 & Si20 & Sidama & Dalle & 1940 \\
\hline 21 & Si21 & Sidama & Dalle & 1940 (Gellawcho-1) \\
\hline 22 & $\mathrm{Si} 22$ & Sidama & Dalle & 1940 \\
\hline 23 & $\mathrm{Si} 23$ & Sidama & Dalle & 1940 (Adameado) \\
\hline 24 & Si24 & Sidama & Dalle & 1940 (Gellawcho-2) \\
\hline 25 & $\mathrm{Si} 25$ & Sidama & Dalle & 1940 (Genticha-5) \\
\hline 26 & Si26 & Sidama & Dalle & 1940 (Genticha-6) \\
\hline 27 & Si27 & Sidama & Dalle & 1940 (Midasho) \\
\hline 28 & $\mathrm{Si} 28$ & Sidama & Dalle & 1940 (Genticha-5) \\
\hline 29 & Si29 & Sidama & Dalle & 1940 (Wendu-5) \\
\hline 30 & $\mathrm{Si} 30$ & Sidama & Dalle & 1940 (Wendu-6) \\
\hline 31 & $\mathrm{Si} 31$ & Sidama & & \\
\hline 32 & Wo1 & Wolita & KedidaGamela & 1970 \\
\hline 33 & Wo2 & Wolita & KedidaGamela & 1970 \\
\hline 34 & Wo3 & Wolita & Damotwoyde & 1780 (Oha-1) \\
\hline 35 & Wo4 & Wolita & Wolita & \\
\hline 36 & Wo5 & Wolita & Wolita & \\
\hline 37 & Wo6 & Wolita & Wolita & \\
\hline 38 & Wo7 & Wolita & Wolita & \\
\hline 39 & Wo8 & Wolita & Wolita & \\
\hline 40 & Wo9 & Wolita & Wolita & \\
\hline 41 & Wo10 & Wolita & Wolita & \\
\hline 42 & Wo11 & Wolita & Damotgalle & 1950 (Gassa) \\
\hline 43 & Wo12 & Wolita & Sodozuria & 1780 (Oha2) \\
\hline 44 & Wo13 & Wolita & Sodozuria & 1780 (Oha3) \\
\hline 45 & Wo14 & Wolita & Sodozuria & 1780 (Oha4) \\
\hline 46 & Wo15 & Wolita & Sodozuria & 1850 (Chocha-1) \\
\hline 47 & Wo16 & Wolita & Sodozuria & 1850 (Chocha-2) \\
\hline 48 & Wo17 & Wolita & Wolita & \\
\hline 49 & Wo18 & Wolita & Wolita & \\
\hline 50 & Wo19 & Wolita & KedidaGamela & 1970 \\
\hline 51 & Wo20 & Wolita & KedidaGamela & 1970 \\
\hline 52 & Wo21 & Wolita & KedidaGamela & 1970 \\
\hline
\end{tabular}




\begin{tabular}{|c|c|c|c|c|}
\hline SN & Accession number & Zone & Location & Altitude(Local name) \\
\hline 53 & Wo22 & Wolita & Alabanatembar & 1630 \\
\hline 54 & Wo23 & Wolita & Alabanatembar & 1630 \\
\hline 55 & Wo24 & Wolita & Alabanatembar & 1630 \\
\hline 56 & Wo25 & Wolita & Wolita & \\
\hline 57 & Wo26 & Wolita & Wolita & \\
\hline 58 & Wo27 & Wolita & Damotwoyde & 1780 (Wiyacha-1) \\
\hline 59 & Wo28 & Wolita & Damotwoyde & 1780 (Wiyacha-2) \\
\hline 60 & Wo29 & Wolita & Wolita & \\
\hline 61 & Wo30 & Wolita & Damotwoyde & 1780 (Oha-5) \\
\hline 62 & Wo31 & Wolita & Damotwoyde & 1780 (Oha-6) \\
\hline 63 & Wo32 & Wolita & Wolita & \\
\hline 64 & Wo33 & Wolita & Kokate & Chaye Woncharo \\
\hline 65 & Wo34 & Wolita & Damotgalle & 2220 (Arkiya) \\
\hline 66 & Wo35 & Wolita & Areka & 1870 (Gaffella-1) \\
\hline 67 & Wo36 & Wolita & Areka & 1870 (Gaffella-2) \\
\hline 68 & Wo37 & Wolita & Areka & 1870 (Gaffella-3) \\
\hline 69 & Wo38 & Wolita & Wolita & \\
\hline 70 & Wo39 & Wolita & Wolita & \\
\hline 71 & Wo40 & Wolita & Areka & 1870 (Gaffela-4) \\
\hline 72 & Wo41 & Wolita & Areka & 1870 (Gaffela-5) \\
\hline 73 & Wo42 & Wolita & Wolita & \\
\hline 74 & Wo43 & Wolita & Wolita & (Wancharo-1) \\
\hline 75 & Wo44 & Wolita & Wolita & (Wancharo-2) \\
\hline 76 & Wo45 & Wolita & Wolita & (Wancharo-3) \\
\hline 77 & Wo46 & Wolita & Wolita & - \\
\hline 78 & Wo47 & Wolita & Damotgalle & 2220 (Arkiya-1) \\
\hline 79 & Wo48 & Wolita & Damotgalle & 2220 (Arkiya-2) \\
\hline 80 & Wo49 & Wolita & Wolita & - \\
\hline 81 & Wo50 & Wolita & Damotwoyde & 2220 (Wiyacha-1) \\
\hline 82 & Wo51 & Wolita & Damotwoyde & 2220 (Wiyacha-2) \\
\hline 83 & Wo52 & Wolita & Damotwoyde & 2220 (Wiyacha-3) \\
\hline 84 & Wo53 & Wolita & Wolita & - \\
\hline 85 & Eg1 & EastWolega & Diga & 1650 (Lalo-1) \\
\hline 86 & $\mathrm{Eg} 2$ & EastWolega & Diga & 1650 (Lalo-2) \\
\hline 87 & Eg3 & EastWolega & Diga & 1650 (Lalo-3) \\
\hline 88 & Eg4 & EastWolega & Diga & 1650 (Msreta-1) \\
\hline 89 & Eg5 & EastWolega & Diga & 1650 (Msreta-2) \\
\hline 90 & Eg6 & EastWolega & Diga & 1650 (Msereta-3) \\
\hline 91 & Eg7 & EastWolega & Sasiga & 1750 (Sasiga-1) \\
\hline 92 & Eg8 & EastWolega & Sasiga & 1750 (Sasiga-2) \\
\hline 93 & $\mathrm{Eg} 9$ & EastWolega & Sasiga & 1750 (Sasiga-3) \\
\hline 94 & Eg10 & EastWolega & Diga & 1650 (Dhoknuma-1) \\
\hline 95 & Eg11 & EastWolega & Diga & 1650 (Dhoknuma-2) \\
\hline 96 & Eg12 & EastWolega & Diga & 1650 (Dhokuma-3) \\
\hline 97 & Arl & ArekaRC & $\mathrm{ARC}$ & 1780 \\
\hline 98 & Ar2 & ArekaRC & $\mathrm{ARC}$ & 1780 \\
\hline 99 & Ar3 & ArekaRC & ARC & 1780 \\
\hline 100 & Ar4 & ArekaRC & ARC & 1780 \\
\hline 101 & $\operatorname{Ar} 5$ & ArekaRC & ARC & 1780 \\
\hline 102 & Ar6 & ArekaRC & $\mathrm{ARC}$ & 1780 \\
\hline 103 & $\operatorname{Ar} 7$ & ArekaRC & ARC & 1780 \\
\hline 104 & Ar8 & ArekaRC & ARC & 1780 \\
\hline 105 & Ar9 & ArekaRC & ARC & 1780 \\
\hline 106 & Ar10 & ArekaRC & ARC & 1780 \\
\hline 107 & Ar11 & ArekaRC & ARC & 1780 \\
\hline 108 & Ar12 & ArekaRC & ARC & 1780 \\
\hline 109 & Ar13 & ArekaRC & ARC & 1780 \\
\hline 110 & Ar14 & ArekaRC & $\mathrm{ARC}$ & 1780 \\
\hline 111 & Ar15 & ArekaRC & ARC & 1780 \\
\hline
\end{tabular}




\begin{tabular}{|c|c|c|c|c|}
\hline SN & Accession number & Zone & Location & Altitude(Local name) \\
\hline 112 & Ar16 & ArekaRC & ARC & 1780 \\
\hline 113 & Ar17 & ArekaRC & $\mathrm{ARC}$ & 1780 \\
\hline 114 & Ar18 & ArekaRC & $\mathrm{ARC}$ & 1780 \\
\hline 115 & Ar19 & ArekaRC & $\mathrm{ARC}$ & 1780 \\
\hline 116 & $\operatorname{Ar} 20$ & ArekaRC & $\mathrm{ARC}$ & 1780 \\
\hline 117 & $\operatorname{Ar} 21$ & ArekaRC & $\mathrm{ARC}$ & 1780 \\
\hline 118 & Ar22 & ArekaRC & $\mathrm{ARC}$ & 1780 \\
\hline 119 & Ar23 & ArekaRC & $\mathrm{ARC}$ & 1780 \\
\hline 120 & Ar24 & ArekaRC & $\mathrm{ARC}$ & 1780 \\
\hline 121 & $\operatorname{Ar} 25$ & ArekaRC & $\mathrm{ARC}$ & 1780 \\
\hline 122 & $\operatorname{Ar} 26$ & ArekaRC & $\mathrm{ARC}$ & 1780 \\
\hline 123 & $\operatorname{Ar} 27$ & ArekaRC & $\mathrm{ARC}$ & 1780 \\
\hline 124 & Ar28 & ArekaRC & ARC & 1780 \\
\hline 125 & $\operatorname{Ar} 29$ & ArekaRC & ARC & 1780 \\
\hline 126 & $\operatorname{Ar} 30$ & ArekaRC & $\mathrm{ARC}$ & 1780 \\
\hline 127 & $\operatorname{Ar} 31$ & ArekaRC & $\mathrm{ARC}$ & 1780 \\
\hline 128 & $\operatorname{Ar} 32$ & ArekaRC & $\mathrm{ARC}$ & 1780 \\
\hline 129 & $\operatorname{Ar} 33$ & ArekaRC & $\mathrm{ARC}$ & 1780 \\
\hline 130 & $\operatorname{Ar} 34$ & ArekaRC & $\mathrm{ARC}$ & 1780 \\
\hline 131 & Ga1 & GamoGofa & Gofa & 1340 \\
\hline 132 & $\mathrm{Ga} 2$ & GamoGofa & Kucha & 1500 (Bunne-3) \\
\hline 133 & $\mathrm{Ga} 3$ & GamoGofa & Bonke & 2070 (Kemba-1) \\
\hline 134 & Ga4 & GamoGofa & Bonke & 2070 (Kemba-2) \\
\hline 135 & $\mathrm{Ga} 5$ & GamoGofa & Bonke & 2070 (Kemba-3) \\
\hline 136 & Ga6 & GamoGofa & Arbaminch & 1140 (Hatiya-2) \\
\hline 137 & $\mathrm{Ga} 7$ & GamoGofa & Breda & 1655 (Bunne-2) \\
\hline 138 & $\mathrm{Ga} 8$ & GamoGofa & Gofa & 1340 (Tolla-1) \\
\hline 139 & Ga9 & GamoGofa & Gofa & 1340 (Tolla-2) \\
\hline 140 & Ga10 & GamoGofa & Gofa & 1340 (Tolla-3) \\
\hline 141 & Ga11 & GamoGofa & Gofa & 1340 (Tolla-3) \\
\hline 142 & Ga12 & GamoGofa & Gofa & 1340 (Bunne-1) \\
\hline 143 & Ga13 & GamoGofa & Gofa & 1340 (Bunne-2) \\
\hline 144 & Ga14 & GamoGofa & Gofa & 1340 \\
\hline 145 & Ga15 & GamoGofa & Gofa & 1340 \\
\hline 146 & Ga16 & GamoGofa & Gofa & 1340 \\
\hline 147 & Ga17 & GamoGofa & Gofa & 1340 \\
\hline 148 & Ga18 & GamoGofa & Gofa & 1340 \\
\hline 149 & Ga19 & GamoGofa & Gofa & 1340 \\
\hline 150 & $\mathrm{Ga} 20$ & GamoGofa & Gofa & 1340 \\
\hline 151 & $\mathrm{Ga} 21$ & GamoGofa & Bonke & 1540 (Arfa-1) \\
\hline 152 & $\mathrm{Ga} 22$ & GamoGofa & Bonke & 1540 (Arfa-2) \\
\hline 153 & $\mathrm{Ga} 23$ & GamoGofa & Bonke & 1540 (Arfa-3) \\
\hline 154 & $\mathrm{Ga} 24$ & GamoGofa & Bonke & 1540 (Arfa-4) \\
\hline 155 & $\mathrm{Ga} 25$ & GamoGofa & Gofa & 1340 \\
\hline 156 & $\mathrm{Ga} 26$ & GamoGofa & Gofa & 1340 \\
\hline 157 & $\mathrm{Ga} 27$ & GamoGofa & Kucha & 1500 (Bunne-3) \\
\hline 158 & $\mathrm{Ga} 28$ & GamoGofa & Gofa & 1340 (Tolla) \\
\hline 159 & Ga29 & GamoGofa & Arbaminch & 1140 (Bunne-3) \\
\hline 160 & $\mathrm{Ga} 30$ & GamoGofa & Arbaminch & 1140 (Bunne-4) \\
\hline 161 & Ga31 & GamoGofa & Arbaminch & 1140 (Bunne-5) \\
\hline 162 & Ga32 & GamoGofa & Arbaminch & 1140 (Bunne-6) \\
\hline 163 & Ga33 & GamoGofa & Bonke & 1540 (Kemba-1) \\
\hline 164 & Ga34 & GamoGofa & Bonke & 1540 (Kemba-2) \\
\hline 165 & Ga35 & GamoGofa & Bonke & 1540 (Kemba-3) \\
\hline 166 & Ga36 & GamoGofa & Gofa & 1340 \\
\hline 167 & Gd1 & Gedio & Gedio & 1590 \\
\hline 168 & $\mathrm{Gd} 2$ & Gedio & Gedio & 1590 \\
\hline 169 & $\mathrm{Gd} 3$ & Gedio & Gedio & 1590 \\
\hline 170 & $\mathrm{Gd} 4$ & Gedio & Gedio & 1590 \\
\hline
\end{tabular}




\begin{tabular}{|c|c|c|c|c|}
\hline SN & Accession number & Zone & Location & Altitude(Local name) \\
\hline 171 & Gd5 & Gedio & Gedio & 1590 \\
\hline 172 & Gd6 & Gedio & Gedio & 1770 (Ganticho1) \\
\hline 173 & Gd7 & Gedio & Gedio & 1770 (Ganticho2) \\
\hline 174 & Gd8 & Gedio & Wonago & 1770 \\
\hline 175 & Gd9 & Gedio & Wonago & 1770 \\
\hline 176 & Gd10 & Gedio & Wonago & 1770 \\
\hline 177 & Gd11 & Gedio & Wonago & 1770 \\
\hline 178 & Gd12 & Gedio & Wonago & 1770 \\
\hline 179 & Ib1 & Kefa & Kefa & 1600 \\
\hline 180 & $\mathrm{Ib} 2$ & Kefa & Kefa & 1600 \\
\hline 181 & Ib3 & Kefa & Kefa & 1600 \\
\hline 182 & $\mathrm{Ib} 4$ & Kefa & Kefa & 1600 \\
\hline 183 & $\mathrm{Ib} 5$ & Kefa & Kefa & 1600 \\
\hline 184 & Ib6 & Kefa & Kefa & 1600 \\
\hline 185 & $\mathrm{~K} 1$ & Kembata & Hadero & 1140 \\
\hline 186 & K2 & Kembata & Hadero & 1140 (Makawa-1 \\
\hline 187 & K3 & Kembata & Hadero & 1140 (Makawa-2) \\
\hline 188 & $\mathrm{~J} 1$ & $\mathrm{JRC}$ & JRC & 1780 \\
\hline 189 & $\mathrm{~J} 2$ & JRC & JRC & 1780 \\
\hline 190 & $\mathrm{~J} 3$ & JRC & JRC & 1780 \\
\hline 191 & $\mathrm{~J} 4$ & JRC & JRC & 1780 \\
\hline 192 & $\mathrm{~J} 5$ & JRC & JRC & 1780 \\
\hline 193 & $\mathrm{~J} 6$ & JRC & JRC & 1780 \\
\hline 194 & $\mathrm{~J} 7$ & JRC & JRC & 1780 \\
\hline 195 & $\mathrm{~J} 8$ & $\mathrm{JRC}$ & JRC & 1780 \\
\hline 196 & J9 & JRC & JRC & 1780 \\
\hline 197 & $\mathrm{~J} 10$ & JRC & JRC & 1780 \\
\hline 198 & $\mathrm{~J} 11$ & JRC & JRC & 1780 \\
\hline 199 & $\mathrm{~J} 12$ & JRC & JRC & 1780 \\
\hline 200 & $\mathrm{~J} 13$ & JRC & JRC & 1780 \\
\hline 201 & $\mathrm{~J} 14$ & $\mathrm{JRC}$ & JRC & 1780 \\
\hline 202 & Dal & Dauro & Konta & 1900 (Gebiche-1) \\
\hline 203 & $\mathrm{Da} 2$ & Dauro & Konta & 1900 (Gebiche-2) \\
\hline 204 & Da3 & Dauro & Konta & 1900 (Gebiche-3) \\
\hline 205 & $\mathrm{Da} 4$ & Dauro & Konta & 1900 (Gebiche-4) \\
\hline 206 & $\mathrm{Da} 5$ & Dauro & Mareka & 1580 (Dorsita-1) \\
\hline 207 & $\mathrm{Da} 6$ & Dauro & Mareka & 1580 (Dorsita-2) \\
\hline 208 & $\mathrm{Da} 7$ & Dauro & Mareka & 1580 (Dorsita-3) \\
\hline 209 & $\mathrm{Da} 8$ & Dauro & Mareka & 1580 (Dorsita-4) \\
\hline 210 & Da9 & Dauro & Mareka & 1580 (Dorsita-5) \\
\hline
\end{tabular}

\section{Result and Discussion}

Analysis of Molecular Variance revealed a significant difference among population based on their geographical location (Table 4).

Table 4. Analysis of Molecular Variance (AMOVA) showing the distribution of genetic diversity within and among 210 yam land races.

\begin{tabular}{lllllll}
\hline Source & Df & SS & MS & Est. Var. & \% & P-Value \\
\hline Among Pops & 9 & 79.163 & 8.796 & 0.022 & 0.004 \\
Among Indiv & 200 & 1586.602 & 7.933 & 2.487 & 0.457 & $45 \%$ \\
Within Indiv & 210 & 621.500 & 2.960 & 2.960 & $54 \%$ & 0.459 \\
Total & 419 & 2287.264 & & 5.468 & $100 \%$ \\
\hline
\end{tabular}

\subsection{Yam (Dioscorea Species) Diversity Within Geographical Locations}

Genetic diversity was estimated for location using
Jaccard's similarity coefficient, Neis' Gene diversity, Polymorphism percentage and Shanon Weaver diversity index (Table 5). The total percentage of polymorphic loci ranged from $98.53 \%$ (Wolita) to $94.14 \%$ (Gamogofa). Gene 
diversity was highest $(0.38)$ within accessions collected from Gedio followed by (0.35) with in accessions collected from Jima and Gamogofa. Accessions from Wolega and Kembata with (0.29) revealed lower Gene diversity. Shannon's information index (I) also showed the existence of high genetic variation within the accessions of Gedio, Wolita and Gamogofa. The mean of Jacard's similarity coefficient for comparisons among accessions within locations was 0.18 (Table 5).

\subsection{Yam (Dioscorea Species) Diversity Among Geographical Locations}

The genetic distance was computed based on 10 yam geographical location where originally the germplasms were collected. The highest genetic distance was encountered between Gamogofa and Wolita (0.98), followed by Gagomofa and Sidama (0.96) and Gamogofa and ARC (0.96). Generally lower genetic distances were observed between Kembata to all other locations (Table 6).

Table 5. Summary of Gene Diversity Parameters with in different geographical locations of 210 yam (Dioscorea species) landraces from Ethiopia.

\begin{tabular}{|c|c|c|c|c|c|}
\hline Zone & No of entries & $\mathbf{P \%}$ & Jaccard'sCoefficient & Nei's geneDiversity (h) & Shannon's InformationIndex $(I)$ \\
\hline Sidama & 31 & 89.71 & 0.14 & 0.28 & $0.427 \pm 0.026$ \\
\hline Wolita & 53 & 98.53 & 0.20 & 0.30 & $0.458 \pm 0.020$ \\
\hline Wolega & 12 & 82.35 & 0.17 & 0.29 & $0.399 \pm 0.028$ \\
\hline $\mathrm{ARC}$ & 34 & 92.65 & 0.19 & 0.32 & $0.459 \pm 0.025$ \\
\hline Gamogofa & 36 & 94.12 & 0.24 & 0.35 & $0.481 \pm 0.022$ \\
\hline Gedio & 12 & 82.35 & 0.20 & 0.38 & $0.457 \pm 0.036$ \\
\hline Kefa & 6 & 75.00 & 0.09 & 0.34 & $0.417 \pm 0.31$ \\
\hline Kembata & 6 & 47.06 & 0.21 & 0.29 & $0.295 \pm 0.039$ \\
\hline Jima & 11 & 80.88 & 0.17 & 0.35 & $0.444 \pm 0.030$ \\
\hline Dauro & 9 & 79.41 & 0.16 & 0.31 & $0.414 \pm 0.029$ \\
\hline Mean & & 82.21 & 0.18 & 0.32 & $0.414 \pm 0.029$ \\
\hline $\mathrm{SD}$ & & & & 0.13 & 0.16 \\
\hline SE & 4.56 & & & & \\
\hline Total Number Polymorphic Loci & 208 & & & & \\
\hline
\end{tabular}

Table 6. Neis' genetic distance measure of Ethiopian yam (Dioscorea species) on the bases of 10 geographical sites.

\begin{tabular}{|c|c|c|c|c|c|c|c|c|c|}
\hline & Sidama & Wolita & Wolega & ARC & Gamogofa & Gedio & kefa & Kembata & Jima \\
\hline \multicolumn{10}{|l|}{ Sidama } \\
\hline Wolita & 0.95 & & & & & & & & \\
\hline Wolega & 0.92 & 0.91 & & & & & & & \\
\hline $\mathrm{ARC}$ & 0.95 & 0.97 & 0.91 & & & & & & \\
\hline Gamogofa & 0.96 & 0.98 & 0.92 & 0.96 & & & & & \\
\hline Gedio & 0.92 & 0.91 & 0.91 & 0.91 & 0.92 & & & & \\
\hline Kembata & 0.68 & 0.66 & 0.70 & 0.67 & 0.68 & 0.70 & 0.67 & & \\
\hline Jima & 0.93 & 0.90 & 0.88 & 0.92 & 0.92 & 0.90 & 0.83 & 0.71 & \\
\hline Dauro & 0.92 & 0.89 & 0.91 & 0.91 & 0.92 & 0.93 & 0.82 & 0.69 & 0.88 \\
\hline
\end{tabular}

\subsection{Cluster Analysis}

A dendrogram was constructed with a UPGMA cluster analysis based on Nei's genetic distance among 10 sites. The dendrogram based on SSR markers distinguished 210 yam landraces in to eight distinctly cluster groups (Figure 2).

Comparison of yam landraces within the same geographical zone revealed that most of yam population from the same origin falls in one or two cluster groups and had the range of similarity coefficient from 0.09 to 0.24 (Table 5). This indicates that the presence of associations between geographical locations and genetic distance.

The second larger cluster group (Cluster 2) consisted of 40, 10, 7, 6, 4, 6 and 11 yam landraces from Gamogofa, Gedio, Jima, Kefa, Kembata, Wolita zones and Areka Research Center. Group 1 is the smallest whereas cluster 6 is the largest group consists of 1 and 52 yam landraces respectively from Wolita zone. Group 3 and 5 is composed of 14 and 10 yam land races from Sidama Zone. Group 4 is made of 11 and 3 yam accessions from Sidama and Dauro zones respectively. Group 8 is composed of 18 yam accessions of Areka Research Center and Group 9 is the smaller group next to cluster 1 which composed of 5 yam accessions where each 2 and 1 accession from Jima and Dauro and, Sidama Zones respectively.

A three dimensional PCO was done using Nei's genetic distances among 10 geographical sites (Figure 3). The first three principal coordinates accounted for $29 \%$ of the total variation among geographical sites which PCO-1 accounts for $14 \%$, PCO-2 for $8 \%$ and PCO-3 for $7 \%$. As it is observed from the 3D graph (Figure 3), yam germplasms from Gamogofa zone isolated from most of the yam germplasm of other localities. 


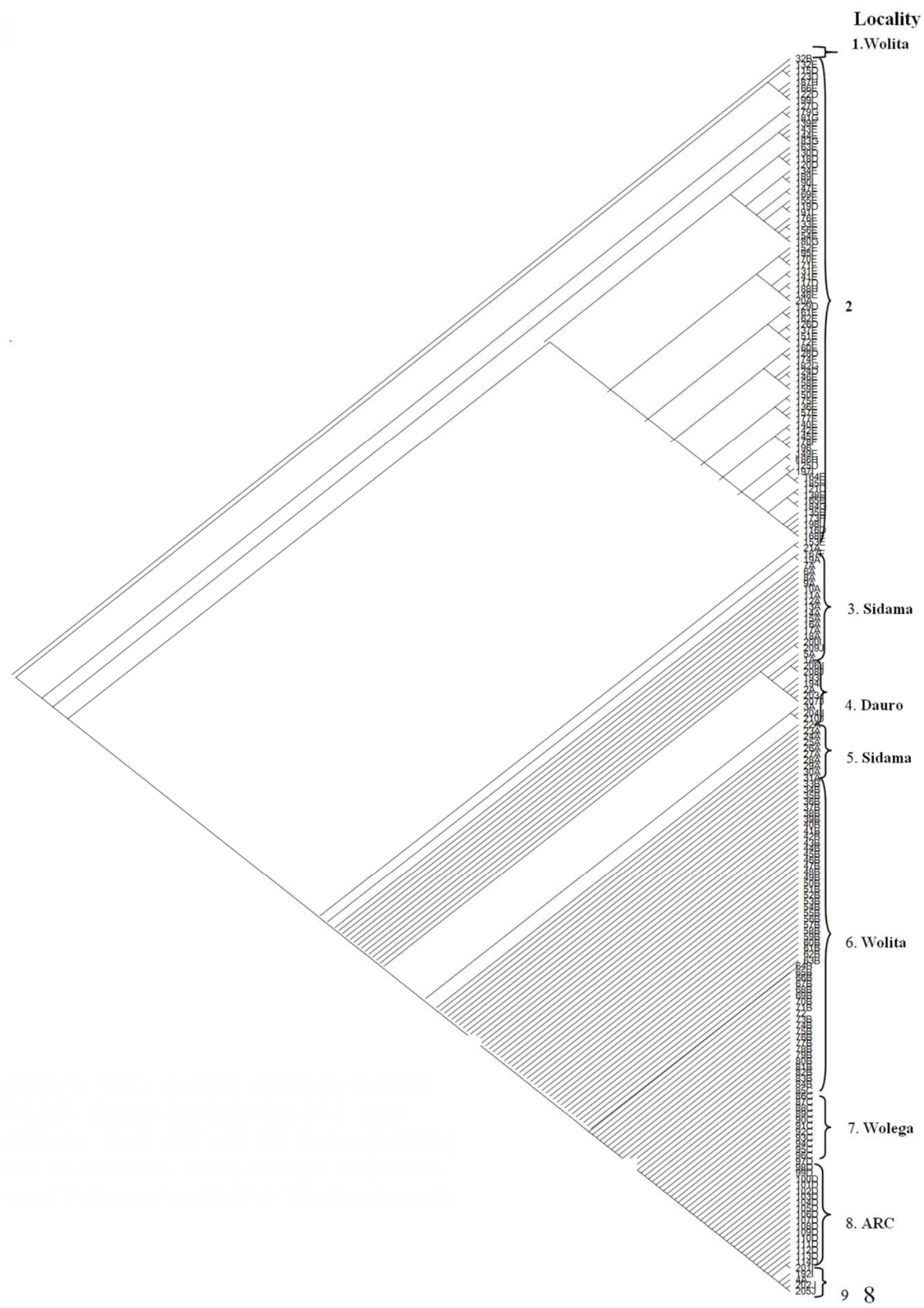

Figure 2. Nei's genetic distance derived UPGMA cluster analysis for 210 yam landraces from SW and SE major growing areas of Ethiopia using Simple Sequence Repeat Polyacrylamide Gel-Electrophoresis. 


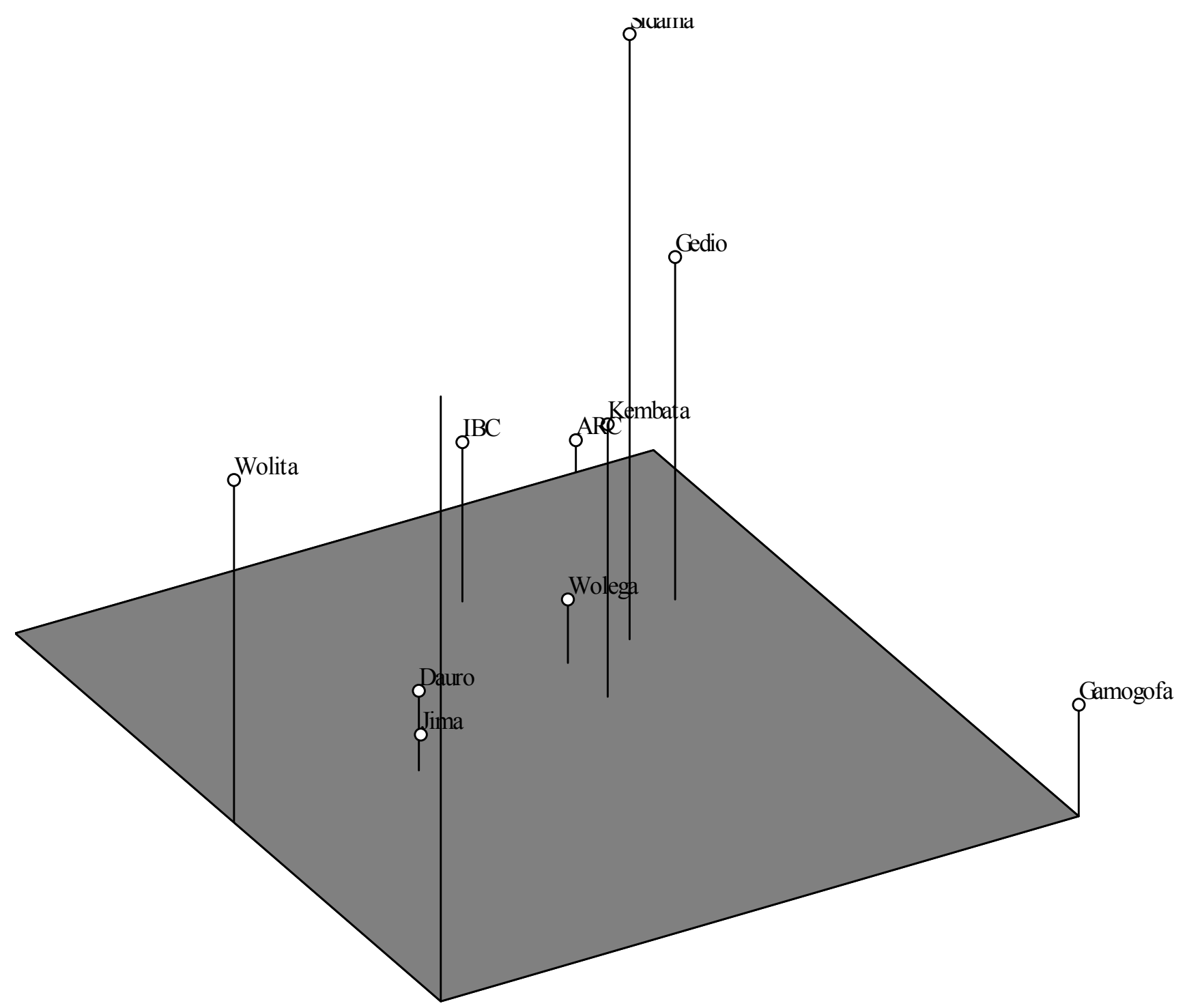

Figure 3. Three diamentional plot for two hundred ten yam landraces of 10 major growing areas of SW and SE Ethiopia using Simple Sequence Repeat Polyacrylamide Gel Electrophoresis.

\section{Discussions}

Understanding the molecular basis of essential biological phenomena in plants is crucial for effective conservation, management, and efficient utilization of plant genetic resources (Linda, et al, 2009). A research of published DNA sequences reveals that Microsatellites (SSRs) are shorter (1-6 base long) which are highly abundant and randomly distributed throughout the eukaryotic genomes [12] and [13]. They are the preferred molecular marker system for analysis in plant genetics and ecology [14].

The present study showed highest polymorphism among Ethiopian yam landraces. The polymorphism percentage among populations of different sites ranged from $98.53 \%$ (Wolita) to $47.06 \%$ (Kembata) with a mean value of 82.21 . This highest level of polymorphism of SSR techniques indicates that this molecular marker is very useful for yam molecular characterization studies.
The un-weighed neighbor joining tree obtained using UPGMA analysis revealed nine distinct clustering groups (Figure 2). It is recommended that geographic diversity may be used as an index of genetic diversity in parental selection [15]. This shows that the power of microsatellite markers for genotyping yam (Dioscorea spp) populations. Microsatellite markers used in this study indicated presence of geographical pattern of diversity within Ethiopian yam landraces apart from cluster group 2. Cluster 2 grouping is in line with the genetic variation result of AMOVA which was very low among geographical sites (Table 4). Earlier, it is also reported similar genetic variation results of AMOVA [16]. An additional reason for cluster 2 grouping may be due to yam germplasms distribution across different locations through market. There is an agreement of this result which stated that yam accessions from different countries were grouped together to one cluster without having any relationship and relatedness of these accessions and their geographical origin [17]. 
Ethiopian yam genotyping based on this molecular marker showed, landraces of Gamogofa exhibits high genetic distance to the majority of yam landraces of other localities and similarly yam germplasms of wolita gave high genetic distance to Wolega, Gamogofa and Areka research center. So it indicates importance of giving consideration to these distantly related populations of Gamogofa, Wolita and Wolega during improvement of the crop through breeding Whereas Kembata landraces revealed less genetic distance towards most of other location. Additionally, high genetic variability was obtained with in Jima and Gedio yam landraces. Accordingly, it is vital to observe these germplasms during selection activities to increase yam production and productivity.

A three diamentional plots (Figure 3) shows the first three principal component scores cumulatively explains $39 \%$ of the total variation which indicated that the genetic variability of Ethiopian yam landraces. This result is comparable to $34 \%$ of total variation research report on the characterization of West Africa yam (Dioscorea alata) using SSR [18].

\section{Conclusion and Recommendations}

The existence of genetic variability is the bases of any plant breeding and improvement activities. The present study showed highest polymorphism $99.05 \%$ among Ethiopian yam landraces. The obtained highest level of polymorphism with SSR techniques indicates that, this molecular marker is vital and powerful for yam molecular characterization studies.

There was clear relationship of genetic distance to that of geographical distance to most of clustering groups. Hence, it is possible to use yam landraces with heritable and desirable characters from distantly related localities as a source of parental material in yam breeding program.

The landraces of Gamogofa exhibits high genetic distance to the majority of yam landraces of other localities and similarly yam germplasms of wolita gave high genetic distance to Wolega, and Gamogofa, so it will be important to consider these distantly related populations of Gamogofa, Wolita, and Wolega in yam breeding for its' genetic gain. Kembata land races revealed less genetic distance towards other location.

High genetic variability was obtained with in Gamogofa, Wolita, Jima and Gedio yam landraces. Accordingly, it is vital to observe these locations during collection and selection of elite clones in yam improvement programs.

\section{References}

[1] IBC, (2008). Ethiopia; Second Country Report on the State of PGRFA to FAO Addis Ababa, Ethiopia.

[2] Kay, D. (1987). Root Crops Tropical Development and Research Institute. London.

[3] Edossa Etissa (1998). Yams: exploration, collection and evaluation. News letter of Ethiopia Agricultural Research Organization. Vol. 13, No. 2.
[4] Miège, J., and Demissew Sebsebe (1997). Dioscoreaceae. In: Flora of Ethiopia an Eriterea (Edwards, S., Sebsebe Demissew and Hedberg, I., eds.). A joint publication of the National Herbarium, Biology Department, AAU, Ethiopia and the depar-tment of Systematic Botany, Uppsala University, Sweden.

[5] Borsch, T., Hilu, K. W., Quandt, D., Wilde, V., Neinhuis, C. and Barthlott, W. (2003). Noncoding plastid trnT-trnF sequences reveal a well resolved phylogeny of basal angiosperms. J. Evol. Biol. 16: 558-576.

[6] Rous, K. (1995). Optimization and troubleshooting in PCR, in PCR primer (Diefferbach, C. W. and Dveksler, G. S eds.), CSH press, New York.

[7] Peakall, R., and Smouse, P. (2006) GenAlEx 6: Genetic analysis in Excel Population genetic software Mol. Ecol. Notes 6, 288-295.

[8] Yeh, F., Boyle, T. (1997). Population genetic analysis of codominant markers and quantitative traits. Belgian Journal of Botany, 129: 157.

[9] Pavlicek, A., Hrda, S. and Flegr, J. (1999). Free tree free ware program for construction of phylogenetic trees on the basis of distance data and bootstrap/Jack Knife analysis of the tree robustness. Application in the RAPD analysis of genus Frenkelia. Folia. Biologica. 45: 97-99.

[10] Sneath, P, and Sokal, R. (1973). Principles of Numerical Taxonomy Freeman San Francisco.

[11] Pavlicek Nei's, M. (1978). Estimation of average heterozygosis and genetic distance from a small number of individuals. Genetics, 3 (89). 583-590.

[12] Linda, M., Arshiya, N., and Mario, A. (2009). Assessing Plant Genetic Diversity by Molecular Tools. Department of Aerobiology and Agro chemistry, Tuscia University, Italy.

[13] Morgante, M., and Olivier, A. (1993). PCR-amplified microsatellites as markers in plant genetics. Plant J. 3, 175182.

[14] Morgante, M., Hanafey, H., and Powell, W. (2002). Microsatellites are preferentially associated with non repetitive DNA in plant genome. Nature Genet 30: 194-200 Tropical Agriculture (Trinidad) 54: 1-13.

[15] Singh, B. D. (2000). Plant Breeding: Principles and Methods. Kalyani Publishers, New Delhi-Ludhiana.

[16] Muluneh, Tamru. (2006). Assessing Diversity in Yams (Dioscorea spp.) from Ethiopia based on, Morphology, AFLP Markers and Tuber Quality, and Farmers management of landraces.

[17] Obidiegwu, J., Kolesnikova-Allen M., Ene-obong, E., Muoneke, C., and Asiedu, A., (2009). SSR markers reveal diversity in Guinea yam (D. cayenensis/D. rotundata) core set. African Journal of Biotechnology Vol. 8 (12), pp. 2730-2739.

[18] Obidiegwu, E., Asiedu, R., Ene-Obong, E., Muoneke, C, and Kolesnikova, M, (2009). Genetic characterization of some water yam (Dioscorea alata L.) accessions in West Africa with simple sequenc repeats. Journal of Food \& Agriculture. 